\title{
Quantités ingérées et activités alimentaires comparées des ovins, bovins et caprins recevant des fourrages conservés avec ou sans aliment concentré. I. Etude descriptive
}

\author{
JP Dulphy *, B Carle, C Demarquilly \\ avec la collaboration technique de L l'Hôtelier, G Moins et A Ollier \\ INRA-CRZV de Theix, station de recherches sur la nutrition des herbivores, \\ 63122 Ceyrat, France
}

(Reçu le 18 décembre 1989; accepté le 20 mai 1990)

\begin{abstract}
Résumé - Les quantités de matière sèche ingérées, les activités alimentaires (durée d'ingestion, durée unitaire d'ingestion, nombre de repas par jour, durée des grands repas) et les activités méryciques (durée de rumination, durée unitaire de rumination et temps de latence après les grands repas) ont été comparées pour 5 types d'animaux : béliers castrés, brebis taries, chèvres taries, taurillons et vaches laitières. Quatre fourrages ont été distribués : ensilage de maïs, foin précoce, foin tardif et ensilage d'herbe, seuls ou avec $30 \%$ d'aliment concentré.

Les quantités moyennes ingérées par les 5 types d'animaux ont été égales respectivement à 62,7 , $61,6,61,0,79,7$ et $116 \mathrm{~g}$ de $\mathrm{MS} / \mathrm{kg} \mathrm{P}^{0,75}$. Les durées journalières d'ingestion des fourrages ont varié de $214 \mathrm{~min} / \mathrm{j}$ pour les béliers à 337 pour les vaches, les durées unitaires de $3,67 \mathrm{~min} /\left(\mathrm{g} / \mathrm{kg} \mathrm{P}^{0,75}\right)$ pour les vaches à 5,41 pour les chèvres, le nombre de repas par jour de 6,3 pour les moutons à 9,2 pour les vaches et la durée des grands repas (ceux suivant les 2 distributions de fourrage) de 61 min pour les brebis à 94 pour les vaches.

La variabilité des paramètres caractérisant les activités alimentaires est toujours très élevée : coefficients de variation allant de 12 à $27 \%$.

Lors de chacun des grands repas, les quantités ingérées représentent 30 à $34 \%$ des quantités journalières ingérées.

Les durées journalières de rumination des rations ont été comprises entre $383 \mathrm{~min} / \mathrm{j}$ pour les taurillons et 510 pour les béliers et les vaches, les durées unitaires de rumination des fourrages ont varié de $5,46 \mathrm{~min} /\left(\mathrm{g} / \mathrm{kg} \mathrm{P} \mathrm{P}^{0,75}\right)$ pour les vaches à 10,02 pour les béliers et les temps de latence de 26 min pour les vaches à 65 pour les chèvres.

La durée journalière de mastication est, au même titre que les quantités ingérées, le paramètre dont la variabilité est la plus faible : $10 \%$ en moyenne. Elle varie de $644 \mathrm{~min} / \mathrm{j}$ pour les chèvres à 848 pour les vaches.

L'ensemble des résultats obtenus est discuté et il en ressort les conclusions suivantes : - pour arriver à un niveau d'ingestion donné, les différents individus d'un groupe et les différentes espèces de ruminants peuvent s'organiser très différemment; - l'étude des activités alimentaires est une approche nécessaire pour l'étude de la régulation des quantités ingérées; - les relations entre les différents paramètres enregistrés restent à étudier.
\end{abstract}

ingestion / rumination / ovin / bovin / caprin

* Correspondance et tirés à part. 
Summary - A comparative study of feed intake and feeding activities in sheep, cattle and goats receiving stored forages with or without feed concentrate supplementation. I. A descriptive study. The level of feed intake, feeding activities (daily time spent eating, unitary eating time, number of meals per day, time spent eating large meals) and ruminating-chewing activities (daily time spent ruminating, unitary rumination time, eating pause after large meals) were compared in 5 types of animals: castrated rams, dried ewes, dried goats, young bulls and dairy cows. Four forages were used: maize silage, early hay, late hay and grass silage alone or with $30 \%$ feed concentrate.

The mean levels of intake for the 5 types of animals were $62.7,61.6,61.0,79.7$ and $116 \mathrm{~g} \mathrm{DM} / \mathrm{kg}$ $W^{0.75}$. The daily time spent eating ranged from $214 \mathrm{~min} / \mathrm{d}$ for rams to 337 for cows, the unitary eating time from $3.67 \mathrm{~min} /(\mathrm{g} / \mathrm{kg}$ W0.75) for cows to 5.41 for goats, the number of meals per day from 6.3 for sheep to 9.2 for cows and the time spent eating large meals (after the 2 forage feedings) from 61 min for ewes to 94 for cows. The variability of the parameters characterizing the feeding activities was always very high with variation coefficients from 12 to $27 \%$.

The amount eaten during large meals represented $30-34 \%$ of the daily feed intake.

The daily time spent ruminating ranged from $383 \mathrm{~min} / \mathrm{d}$ for young bulls to 510 for rams and cows, the unitary rumination time ranged from $5.46 \mathrm{~min} /(\mathrm{g} / \mathrm{kg} / \mathrm{W} .75)$ for cows to 10.02 for rams and the eating pause from 26 min for cows to 65 for goats.

The daily time spent chewing, as the amounts ingested, showed the lowest variability, ie on an average $10 \%$. It varied from $644 \mathrm{~min} / \mathrm{d}$ for goats to $848 \mathrm{~min}$ for cows.

Results were discussed and the following conclusions drawn :

- to reach a given level of feed intake, the individuals of a group and the ruminant species may proceed differently;

- studying feeding activities is a necessary approach for in order to study the regulation of feed intake;

- the relationships between the different measured parameters remain to be established.

feed intake / rumination / sheep / cattle / goats

\section{INTRODUCTION}

Les différences de quantités ingérées ef de digestibilité entre les ovins et les caprins d'une part (Brown et Johnson, 1984), entre les ovins et les bovins d'autre part (Chenost et Martin-Rosset, 1985) sont relativement bien connues. Des études se poursuivent d'ailleurs sur ce thème (Huston et al, 1986; Masson, Alrahmoun et Tisserand, 1986; Mc Cabe et Barry, 1988). Les causes des différences constatées ont aussi été largement discutées (Ellis et al, 1988).

En revanche, il existe peu d'études comparatives sur les activités alimentaires d'animaux d'espèces différentes recevant les mêmes fourrages. Or, par rapport aux problèmes posés par la régulation des quantités ingérées, l'étude des activités alimentaires présente deux aspects intéressants. Ces activités sont tout d'abord le reflet de l'action des facteurs internes agissant sur l'appétit et déterminant en particulier le début et la fin des repas. Lorsque la régulation de l'appétit est plutôt d'ordre physique (Conrad et al, 1964), l'observation des activités d'ingestion indique les moments où l'état de replétion du rumen atteint son maximum. Par ailleurs la mastication lors de l'ingestion et surtout de la rumination permettent la réduction de la taille des particules alimentaires nécessaires à leur digestion et à leur transit.

Pour couvrir leurs besoins, les différentes espèces animales peuvent avoir des activités alimentaires organisées différemment. De même, l'ingestion d'aliments 
différents peut engendrer des réactions différentes. Dans les 2 cas, les observations faites peuvent aider à mieux comprendre le rôle des mécanismes mis en jeu pour la régulation des quantités ingérées. Par exemple, ces observations doivent permettre de répondre à certaines questions telles que l'effet de la durée de rumination comme facteur limitant les quantités journalières ingérées ou bien l'importance du ou des grands repas pour ces quantités ingérées.

Pour expliquer les mécanismes de régulation des quantités ingérées d'une espèce à l'autre, l'étude des activités alimentaires et méryciques est donc un moyen privilégié (Dulphy et Faverdin, 1987). C'est pourquoi nous avons, sur des fourrages très différents, entrepris une telle comparaison. $\mathrm{Si}$ quelques études comparatives ont déjà été publiées (Ruckebusch et Bost, 1963; Geoffroy, 1974; Dulphy et MichaletDoreau, 1983; Dulphy et al, 1984; Focant, 1984 et 1986), il faut reconnaître que les résultats bibliographiques sur le sujet sont rares.

Nous avons alors mis en place une série de comparaisons qui ont porté sur 5 types d'animaux et 4 fourrages. Les animaux comparés ont été des ovins (béliers castrés et femelles taries), des bovins (taurillons et vaches laitières) et des caprins (femelles taries). Les 4 fourrages étaient des ensilages (maïs et herbe) et des foins (un précoce et un tardif) distribués à l'auge, seuls ou avec un aliment concentré ( $30 \%$ de la ration).

Le présent travail donne une description détaillée du protocole expérimental et des résultats issus de ces comparaisons dont une partie a déjà été publiée antérieurement (Dulphy et al, 1979; Carle et Dulphy, 1980; Dulphy et Carle, 1986).

L'analyse des liaisons entre paramètres et le problème posé par la comparaison d'espèces de poids vif très différent feront l'objet d'une autre publication.

\section{MATÉRIEL ET MÉTHODES}

\section{Animaux expérimentaux}

Nous avons utilisé simultanément :

- des béliers adultes castrés de race Texel âgés de 2 à 4 ans (= moutons standard);

- des brebis de race Limousine après leur tarissement, non gestantes, âgées de 4-6 ans et maigres;

- des chèvres de race Alpine, non gestantes et taries, âgées de 5-6 ans;

- des taurillons «broûtards" de race Charolais $x$ Salers ou Limousin $x$ Salers, âgés de 1 an;

- des vaches laitières gestantes, de race Holstein, âgées de 3-9 ans, produisant en moyenne $11 \mathrm{~kg}$ de lait en fin de lactation (en moyenne la $3^{\theta}$ ).

Tous ces animaux ont été adaptés à leur cage avant les mesures. Ils étaient 6 par lot, différents pour chaque fourrage, et traités contre les parasites digestifs avant l'étude de chacun de ces fourrages. Compte tenu de quelques défections en cours d'essai, le nombre moyen d'animaux utilisables par lot a été égal à 5,5.

Pendant les mesures, les chaleurs des brebis ont été évitées au moyen d'éponges vaginales imprégnées de PMSG. Les chèvres n'ont pu être utilisées pour le premier fourrage (ensilage de maïs).

L'âge et le poids vif des animaux utilisés sont donnés dans le tableau $\mathrm{I}$.

\section{Fourrages}

Nous avons étudié 4 fourrages dont la nature et la digestibilité ont été volontairement choisies très différentes :

- un ensilage de maïs (plante entière), récolté au stade pâteux-vitreux, finement haché (5$10 \mathrm{~mm}$ ) additionné d'urée, pour partie lors de la mise en silo et pour partie à l'auge; 
Tableau I. Principales caractéristiques des animaux utilisés.
Tableau II. Caractéristiques fermentaires des ensilages $\left(\mathrm{g} / \mathrm{kg} \mathrm{MS}\right.$ sauf $\mathrm{pH}$ et $\mathrm{NH}_{3}$ ).

\begin{tabular}{lcc}
\hline & $\begin{array}{c}\text { Ensilage } \\
\text { de maïs }\end{array}$ & $\begin{array}{c}\text { Ensilage } \\
\text { d'herbe }\end{array}$ \\
\hline $\mathrm{pH}$ & 3,88 & 4,16 \\
$\mathrm{~N}-\mathrm{NH}_{3} \% \mathrm{~N}$ total & 10,6 & 8,2 \\
Acide lactique & 59 & 86 \\
Acide acétique & 14 & 32 \\
Acide propionique & 0,5 & 2,2 \\
Acide butyrique & 0,1 & 2,8 \\
Alcools & 7,6 & 16,2 \\
& & \\
\hline
\end{tabular}

fourrage avec $30 \%$ de concentré, sauf pour le foin de fléole pour lequel il y a eu inversion entre les 2 rations. II y a donc eu au total 8 périodes expérimentales qui se sont succédées dans le temps.

Afin que l'azote ne soit jamais un facteur limitant de la digestion dans le rumen, les fourrages distribués seuls ont été supplémentés :

- avec $0,6 \%$ d'urée et $0,6 \%$ de minéraux pour l'ensilage de maïs;

- avec $6 \%$ de tourteau d'arachide pour les foins.

L'aliment concentré contenait $25 \%$ de tourteau de soja, $30 \%$ d'orge, $42,5 \%$ de maïs et $2,5 \%$ de minéraux.

Les animaux ont toujours reçu en même temps le même régime, d'abord le fourrage seul puis le

Tableau III. Composition chimique des aliments distribués.

\begin{tabular}{|c|c|c|c|c|c|c|}
\hline & \multirow{2}{*}{$\begin{array}{l}\text { Ensilage } \\
\text { de maïs }\end{array}$} & \multicolumn{2}{|c|}{ Foins de } & \multirow{2}{*}{$\begin{array}{l}\text { Ensilage } \\
\text { d'herbe }\end{array}$} & \multirow{2}{*}{$\begin{array}{c}\text { Aliment } \\
\text { concentré }\end{array}$} & \multirow{2}{*}{$\begin{array}{l}\text { Tourteau } \\
\text { d'Arachide }\end{array}$} \\
\hline & & ray-grass & fléole & & & \\
\hline Teneur en MS $(\mathrm{g} / \mathrm{kg})$ & 340 & 886 & 876 & 185 & 885 & 920 \\
\hline $\begin{array}{l}\text { Teneur en g/kg MS } \\
\text { - cendres } \\
\text { - MAT } \\
\text { - CB } \\
\text { - amidon }\end{array}$ & $\begin{array}{r}53 \\
102 \\
169 \\
384\end{array}$ & $\begin{array}{r}78 \\
83 \\
285 \\
-\end{array}$ & $\begin{array}{r}47 \\
98 \\
369 \\
-\end{array}$ & $\begin{array}{r}96 \\
186 \\
278 \\
-\end{array}$ & $\begin{array}{r}53 \\
211 \\
42 \\
567\end{array}$ & $\begin{array}{r}68 \\
483 \\
125 \\
-\end{array}$ \\
\hline
\end{tabular}


Tous les animaux ont reçu également une complémentation minérale et disposaient à volonté d'eau et de pierre à sel.

Chacune des 8 périodes de l'étude a duré 21 j : 16 j pour mettre les animaux en régime et 5 j de mesures. Les fourrages ont été distribués en 2 repas (à $8 \mathrm{~h}$ et $16 \mathrm{~h}$ ). Dans le cas des rations mixtes, l'aliment concentré a été distribué 30 min avant le fourrage.

Chez les petits ruminants, l'influence du tri a été limitée par le hachage des foins. Pour l'ensemble des animaux, qui étaient nourris à volonté, on a recherché un niveau de refus de l'ordre de $10 \%$.

\section{Mesures}

Les quantités d'aliment offertes ont été pesées chaque jour, ainsi que les refus et les fèces le lendemain matin. Les teneurs en MS ont aussi été mesurées chaque jour, dans des étuves ventilées à $80^{\circ} \mathrm{C}$ pendant 24 à $48 \mathrm{~h}$. Celles des ensilages ont été corrigées pour les pertes à l'étuve (Dulphy et Demarquilly, 1981). La digestibilité in vivo de tous les régimes a été mesurée: elle est donnée, à titre d'information, tableau IV.
Les activités alimentaires et méryciques ont été enregistrées à l'aide d'un ballonnet rempli de mousse et maintenu par un licol en position sous-maxillaire (Ruckebusch, 1963). La lecture des tracés a été réalisée selon la méthode décrite par Dulphy (1971). II en est de même pour le mode d'expression des résultats en durées unitaires. Malheureusement, les enregistrements pour les taurillons n'ont concerné que les 2 premiers fourrages car les animaux supportaient mal leur collier. Lors du dépouillement, les temps passés à ingérer l'aliment concentré n'ont pas été pris en comple car ils étaient très faibles.

En outre, pour tous les animaux sur les 6 premiers régimes, on a mesuré les quantités ingérées lors du grand repas du matin et donc la durée unitaire d'ingestion lors de ce grand repas.

\section{Analyses}

Les constituants de la matière sèche (offert, refus et fèces) ont été analysés de façon systématique sur les aliments étudiés pour chaque période et pour chaque lot d'animaux. Les cendres ont été obtenues après incinération à

Tableau IV. Digestibilité de la matière organique des régimes étudiés (\%).

\begin{tabular}{|c|c|c|c|c|c|}
\hline & Moutons & Brebis & Chèvres & Taurillons & Vaches \\
\hline $\begin{array}{l}\text { Ensilage de maïs } \\
\quad-\text { seul (avec urée) } \\
-+30 \% \text { concentré }\end{array}$ & $\begin{array}{l}71,8 \\
72,5\end{array}$ & $\begin{array}{l}73,3 \\
77,5\end{array}$ & $\begin{array}{l}- \\
-\end{array}$ & $\begin{array}{l}70,0 \\
71,4\end{array}$ & $\begin{array}{l}71,6 \\
74,5\end{array}$ \\
\hline $\begin{array}{l}\text { Foin de ray-grass } \\
\qquad+6 \% T \text { arachide } \\
-+30 \% \text { concentré }\end{array}$ & $\begin{array}{l}67,9 \\
70,7\end{array}$ & $\begin{array}{l}71,1 \\
72,6\end{array}$ & $\begin{array}{l}67,9 \\
72,4\end{array}$ & $\begin{array}{l}73,7 \\
73,2\end{array}$ & $\begin{array}{l}74,6 \\
74,6\end{array}$ \\
\hline $\begin{array}{l}\text { Foin de fléole } \\
\qquad \begin{array}{l}-+6 \% \mathrm{~T} \text { arachide } \\
-+30 \% \text { concentré }\end{array}\end{array}$ & $\begin{array}{l}55,6 \\
60,5\end{array}$ & $\begin{array}{l}56,7 \\
62,8\end{array}$ & $\begin{array}{l}57,8 \\
62,9\end{array}$ & $\begin{array}{l}64,1 \\
67,9\end{array}$ & $\begin{array}{l}63,3 \\
68,4\end{array}$ \\
\hline $\begin{array}{l}\text { Ensilage d'herbe } \\
- \text { seul } \\
-+30 \% \text { concentré }\end{array}$ & $\begin{array}{l}74,0 \\
77,3\end{array}$ & $\begin{array}{l}77,8 \\
79,0\end{array}$ & $\begin{array}{l}74,3 \\
77,9\end{array}$ & $\begin{array}{l}76,4 \\
77,0\end{array}$ & $\begin{array}{l}80,5 \\
80,0\end{array}$ \\
\hline $\begin{array}{l}\text { Coefficient de variation } \\
\text { moyen interindividuel (\%) }\end{array}$ & 2,3 & 2,8 & 2,0 & 3,2 & 1,5 \\
\hline
\end{tabular}


$550{ }^{\circ} \mathrm{C}$, les matières azotées (MAT) par la méthode de Kjeldhal $(\mathrm{N} \times 6,25)$, la cellulose brute par la méthode Weende et l'amidon par la méthode de Thivend et al (1965).

\section{Statistiques}

Les résultats des différents paramètres enregistrés ont été soumis à une analyse de variance par le programme SAS (General Linear Models Procedure).

\section{RÉSULTATS}

\section{Quantités de matière sèche (MS) ingérées}

Les quantités journalières de MS ingérées figurent dans le tableau V. Intra-lot, le coefficient de variation des résultats est égal, en moyenne, à $9,5 \%$. On note la plus forte homogénéité pour les vaches et la plus forte hétérogénéité d'un individu à l'autre pour les brebis.
L'effet du type de fourrage ne nous intéresse pas directement. Relevons simplement que, comme souhaité, le foin de fléole a été relativement mal ingéré $(61,7 \mathrm{~g}$ de $M S / k g ~ P 0,75$, dont $6 \%$ de tourteau) alors que le foin de ray-grass $(75,5 \mathrm{~g}$, dont $6 \%$ de tourteau) et l'ensilage d'herbe $(77,3 \mathrm{~g})$ ont été bien ingérés. L'ensilage de maïs a été intermédiaire, avec une ingestibilité relativement élevée chez les bovins, mais faible pour les béliers castrés.

Les taux moyens de substitution fourrage/concentré ont été égaux à 0,76 pour l'ensilage de maïs, 0,74 pour l'ensilage d'herbe, 0,41 pour le foin de fléole et 0,37 pour le foin de ray-grass, ce en excluant des calculs les chèvres qui n'ont pas reçu l'ensilage de maïs.

Pour l'ensemble des 8 rations étudiées, les quantités ingérées par les béliers castrés et les brebis taries sont très proches (62,7 contre $61,6 \mathrm{~g}$ de MS/kg P0,75). Dans les même conditions, les taurillons ont ingéré $79,7 \mathrm{~g}$ et les vaches laitières $116 \mathrm{~g}$.

Tableau V. Quantités de MS ingérées ( $\mathrm{g} / \mathrm{kg} \mathrm{P0,75}$ ).

\begin{tabular}{|c|c|c|c|c|c|}
\hline & Moutons & Brebis & Chèvres & Taurillons & Vaches \\
\hline $\begin{array}{l}\text { Ensilage de maïs } \\
- \text { seul (avec urée) } \\
-+30 \% \text { concentré }\end{array}$ & $\begin{array}{l}49,8 \\
57,7\end{array}$ & $\begin{array}{l}65,5 \\
63,3\end{array}$ & $\begin{array}{l}- \\
-\end{array}$ & $\begin{array}{l}79,0 \\
84,6\end{array}$ & $\begin{array}{l}113,3 \\
129,2\end{array}$ \\
\hline $\begin{array}{l}\text { Foin de ray-grass } \\
\qquad \begin{array}{l}-+6 \% \mathrm{~T} \text { arachide } \\
-+30 \% \text { concentré }\end{array}\end{array}$ & $\begin{array}{l}62,3 \\
71,2\end{array}$ & $\begin{array}{l}52,5 \\
73,7\end{array}$ & $\begin{array}{l}75,0 \\
76,0\end{array}$ & $\begin{array}{l}76,8 \\
77,4\end{array}$ & $\begin{array}{l}110,8 \\
142,8\end{array}$ \\
\hline $\begin{array}{l}\text { Foin de fléole } \\
\qquad \begin{array}{l}-+6 \% \mathrm{~T} \text { arachide } \\
-+30 \% \text { concentré }\end{array}\end{array}$ & $\begin{array}{l}55,2 \\
66,9\end{array}$ & $\begin{array}{l}48,6 \\
58,5\end{array}$ & $\begin{array}{l}48,3 \\
54,4\end{array}$ & $\begin{array}{l}69,0 \\
76,6\end{array}$ & $\begin{array}{r}87,2 \\
102,0\end{array}$ \\
\hline $\begin{array}{l}\text { Ensilage d'herbe } \\
- \text { seul } \\
-+30 \% \text { concentré }\end{array}$ & $\begin{array}{l}67,4 \\
71,2\end{array}$ & $\begin{array}{l}61,6 \\
68,7\end{array}$ & $\begin{array}{l}57,5 \\
54,6\end{array}$ & $\begin{array}{l}83,9 \\
90,2\end{array}$ & $\begin{array}{l}116,1 \\
126,7\end{array}$ \\
\hline $\begin{array}{l}\text { Coefficient de variation } \\
\text { moyen interindividuel (\%) }\end{array}$ & 9,4 & 13,7 & 9,5 & 8,3 & 6,8 \\
\hline
\end{tabular}


Les taux moyens de substitution ont été égaux à 0,54 pour les béliers, 0,50 pour les brebis, 0,78 pour les taurillons et 0,46 pour les vaches.

Avec les 6 régimes reçus en commun, les chèvres ont ingéré $61 \mathrm{~g}$ de MS/kg $\mathrm{P}^{0,75}$ contre $65,7 \mathrm{~g}$ pour les moutons, valeurs significativement différentes. On note néanmoins des valeurs moyennes très proches pour les fourrages seuls $(60,3$ contre 61,6 g), mais des taux de substitution plus élevés chez les chèvres $(0,89)$ que chez les béliers $(0,54)$ entraînant une baisse plus importante des quantités ingérées chez les chèvres que chez les moutons. Pour terminer, notons que les quantités d'aliments ingérées ont, en moyenne, largement couvert les besoins d'entretien des animaux : $+80 \%$ par rapport à leurs besoins d'entretien pour les béliers, les brebis et les taurillons; $+35 \%$ pour les chèvres; $+150 \%$ pour les vaches laitières.

\section{Activités d'ingestion (tableaux VI, VII, VIII)}

\section{Durée journalière}

Ce paramètre présente une variabilité élevée : coefficients de variation intralot égaux respectivement à $17 \%$ pour les ovins, $15 \%$ pour les chèvres, $14 \%$ pour les taurillons et $12 \%$ pour les vaches.

On peut comparer les fourrages (2 rations à chaque fois) en regroupant les résultats obtenus sur béliers, brebis et vaches. Dans ces conditions, les durées d'ingestion sont faibles pour l'ensilage d'herbe (223 $\mathrm{min} / \mathrm{j})$ et l'ensilage de maïs (230 min/j), intermédiaires pour le foin de ray-grass $(272 \mathrm{~min} / \mathrm{j})$ et élevées pour le foin de fléole (330 min/j).

Pour les 8 régimes, le temps passé à ingérer par les vaches est significativement supérieur à celui passé par les béliers et les brebis, le temps pour ces dernières étant significativement supérieur à celui des béliers (tableau VI). Pour les régimes qui leur étaient communs, les chèvres et les taurillons ont passé respectivement 15 et $27 \%$ de temps en plus que les béliers à ingérer chaque jour (différences significatives), mais 30 et $9 \%$ en moins que les vaches.

En présence d'aliment concentré la durée d'ingestion des fourrages a baissé significativement de $23 \%$.

\section{Durée unitaire}

Ce paramètre présente aussi une très grande variabilité : coefficient de variation

Tableau VI. Activités comparées d'ingestion et de rumination entre les moutons, les brebis et les vaches (8 régimes) - écart type ( ).

\begin{tabular}{|c|c|c|c|c|c|}
\hline \multirow[b]{2}{*}{ Quantités ingérées (g MS/kg P0,75) } & \multicolumn{2}{|c|}{ Moutons } & \multicolumn{2}{|c|}{ Brebis } & Vaches \\
\hline & 62,7 & $(5,9)^{a}$ & 61,6 & $(8,6)^{a}$ & $116,0(7,9)^{b}$ \\
\hline Durée journalière d'ingestion (min) & 214 & $(37)^{a}$ & 240 & $(41)^{b}$ & $(41)^{\mathrm{c}}$ \\
\hline Durée unitaire d'ingestion ( $\mathrm{min} / \mathrm{g} / \mathrm{kg} \mathrm{P}^{0,75}$ ) & 4,21 & $(0,56)^{a}$ & 4,87 & $(1,04)^{b}$ & $3,67(0,53)^{\mathrm{c}}$ \\
\hline Nombre de repas par jour & 6,3 & $(1,7)^{\mathrm{a}}$ & 8,2 & $(1,9)^{b}$ & $9,2 \quad(1,6)^{\mathrm{c}}$ \\
\hline Durée des grands repas (min) & 69 & $(13)^{a}$ & 61 & $(14)^{b}$ & $94 \quad(14)^{c}$ \\
\hline Durée journalière de rumination (min) & 510 & $(67)^{a}$ & 482 & $(57)^{\mathrm{b}}$ & $510 \quad(43)^{a}$ \\
\hline Durée unitaire de rumination ( $\mathrm{min} / \mathrm{g} / \mathrm{kg} \mathrm{P}^{0,75}$ ) & 10,02 & $(1,19)^{\mathrm{a}}$ & 9,71 & $(1,24)^{\mathrm{a}}$ & $5,46(0,44)^{b}$ \\
\hline Temps de latence (min) & 56 & $(24)^{a}$ & 59 & $(21)^{a}$ & $26(12)^{b}$ \\
\hline Durée journalière de mastication (min) & 724 & $(85)^{a}$ & 722 & $(70)^{a}$ & $848(61)^{\mathrm{b}}$ \\
\hline Durée unitaire de mastication ( $\mathrm{min} / \mathrm{g} / \mathrm{kg} \mathrm{P}^{0,75}$ ) & 14,22 & $(1,32)^{\mathrm{a}}$ & 14,57 & $(1,84)^{a}$ & $9,13(0,80)^{b}$ \\
\hline
\end{tabular}


intralot égaux respectivement à $13 \%$ pour les béliers et les taurillons, $14 \%$ pour les vaches, $21 \%$ pour les brebis et $22 \%$ pour les chèvres.

Toujours en ne prenant en compte que 3 catégories d'animaux, les durées unitaires d'ingestion ont été respectivement égales à $3,65 \mathrm{~min} /\left(\mathrm{g} / \mathrm{kg} \mathrm{P}^{0,75}\right)$ pour l'ensilage de maïs, 4,19 pour le foin de raygrass, 6,09 pour le foin de fléole et 3,06 pour l'ensilage d'herbe. II y a donc eu de grosses différences d'un fourrage à l'autre.

D'un type d'animal à l'autre les temps passés à ingérer $1 \mathrm{~g}$ de $\mathrm{MS} / \mathrm{kg} \mathrm{P}^{0,75}$ ont été significativement différents (tableau VI). Par rapport aux béliers, on a observé des valeurs supérieures de $16 \%$ pour les brebis et $34 \%$ pour les chèvres, mais inférieures de $3 \%$ (NS) pour les taurillons et de $13 \%$ pour les vaches.

Lorsque les animaux ont reçu l'aliment concentré, la durée unitaire d'ingestion des fourrages a baissé significativement en moyenne de $8 \%$.

\section{Nombre de repas de fourrage}

Les coefficients de variation intralot du nombre de repas de fourrage par jour va- rient de $17-18 \%$ pour les bovins à $23-24 \%$ pour les brebis et les chèvres et à $27 \%$ pour les béliers castrés.

II existe peu de différences entre les fourrages, les nombres de repas étant respectivement 8,0 pour l'ensilage de maïs, 7,1 pour le foin de ray-grass, 7,3 pour le foin de fléole et 9,2 pour l'ensilage d'herbe.

Ce sont les béliers qui ont fait le moins de repas par jour $(6,3)$. Toutes les autres catégories d'animaux en ont fait plus : augmentations significatives de $30 \%$ pour les brebis, $23 \%$ pour les chèvres, $33 \%$ pour les taurillons et de $46 \%$ pour les vaches.

L'apport de concentré n'a pas modifié significativement le nombre de repas de fourrage par jour (-3\% seulement).

\section{Durée des grands repas}

Les grands repas sont ceux qui suivent les 2 distributions journalières de fourrage. Les coefficents de variation intralot de leur durée sont respectivement égaux à 14,5\% pour les bovins, $19 \%$ pour les béliers, $22 \%$ pour les brebis et les chèvres.

II existe entre les fourrages des différences notables avec des valeurs élevées pour les foins (valeurs identiques et égales en moyenne à $92 \mathrm{~min}$ ), faibles pour l'ensi-

Tableau VII. Activités comparées d'ingestion et de rumination entre les moutons, les brebis et les chèvres (6 régimes) ( 2 foins + ensilage d'herbe) - écart type ( ).

\begin{tabular}{lrrrl}
\hline & Moutons & \multicolumn{2}{c}{ Brebis } & \multicolumn{2}{c}{ Chèvres } \\
& & & & \\
\hline Quantités ingérées (g MS/kg P0,75) & $65,7^{\mathrm{a}}$ & $60,6^{\mathrm{b}}$ & $61,0^{\mathrm{b}}$ & \\
Durée journalière d'ingestion (min) & $214^{\mathrm{a}}$ & $259^{\mathrm{b}}$ & $247^{\mathrm{b}}$ & $(38)$ \\
Durée unitaire d'ingestion (min/g/kg P0,75) & $4,03^{\mathrm{a}}$ & $5,37^{\mathrm{b}}$ & $5,41^{\mathrm{b}}$ & $(1,18)$ \\
Nombre de repas par jour & $6,4^{\mathrm{a}}$ & $9,0^{\mathrm{b}}$ & $7,9^{\mathrm{c}}$ & $(1,9)$ \\
Durée des grands repas (min) & $67^{\mathrm{ab}}$ & $62^{\mathrm{b}}$ & $68^{\mathrm{a}}$ & $(15)$ \\
Durée journalière de rumination (min) & $519^{\mathrm{a}}$ & $494^{\mathrm{a}}$ & $397^{\mathrm{b}}$ & $(59)$ \\
Durée unitaire de rumination (min/g/kg P0,75) & $9,77^{\mathrm{a}}$ & $10,20^{\mathrm{a}}$ & $8,14^{\mathrm{b}}$ & $(1,68)$ \\
Temps de latence (min) & $45^{\mathrm{a}}$ & $43^{\mathrm{a}}$ & $65^{\mathrm{b}}$ & $(28)$ \\
Durée journalière de mastication (min) & $734^{\mathrm{a}}$ & $752^{\mathrm{a}}$ & $644^{\mathrm{b}}$ & $(74)$ \\
Durée unitaire de mastication (min/g/kg Po,75) & $13,79^{\mathrm{a}}$ & $15,57^{\mathrm{b}}$ & $13,19^{\mathrm{a}}$ & $(2,16)$ \\
\hline
\end{tabular}


Tableau VIII. Activités comparées d'ingestion et de rumination entre les moutons, les taurillons et les vaches (4 régimes) (ensilage de maïs et foin de ray-grass) - écart type ( ).

\begin{tabular}{lrrrrr}
\hline & Moutons & Taurillons & Vaches \\
\hline Quantités ingérées (g MS/kg P0,75) & $61,0^{\mathrm{a}}$ & $80,4^{\mathrm{b}}$ & & $124,7^{\mathrm{c}}$ \\
Durée journalière d'ingestion (min) & $223^{\mathrm{a}}$ & $284^{\mathrm{b}}$ & $(38)$ & 311 \\
Durée unitaire d'ingestion (min/g/kg P0,75) & $4,50^{\mathrm{a}}$ & $4,31^{\mathrm{a}}$ & $(0,59)$ & $3,04^{\mathrm{b}}$ \\
Nombre de repas par jour & $5,7^{\mathrm{a}}$ & $7,6^{\mathrm{b}}$ & $(1,3)$ & $9,5^{\mathrm{c}}$ \\
Durée des grands repas (min) & $80^{\mathrm{a}}$ & $88^{\mathrm{b}}$ & $(13)$ & $100^{\mathrm{b}}$ \\
Durée journalière de rumination (min) & $515^{\mathrm{a}}$ & $383^{\mathrm{b}}$ & $(57)$ & $485^{\mathrm{c}}$ \\
Durée unitaire de rumination (min/g/kg P0,75) & $10,48^{\mathrm{a}}$ & $5,88^{\mathrm{b}}$ & $(0,85)$ & $4,76^{\mathrm{c}}$ \\
Temps de latence (min) & $66^{\mathrm{a}}$ & $47^{\mathrm{b}}$ & $(18)$ & $35^{\mathrm{c}}$ \\
Durée journalière de mastication (min) & $738^{\mathrm{a}}$ & $667^{\mathrm{b}}$ & $(72)$ & $796^{\mathrm{c}}$ \\
Durée unitaire de mastication (min/g/kg P0,75) & $14,97^{\mathrm{a}}$ & $10,20^{\mathrm{b}}$ & $(1,10)$ & $7,80^{\mathrm{c}}$ \\
\hline
\end{tabular}

lage d'herbe (44 $\mathrm{min})$ et intermédiaires pour l'ensilage de maïs (70 min).

Les 3 types de petits ruminants donnent des résultats peu différents $(66 \mathrm{~min}$ en moyenne). Ceux des taurillons et des vaches sont significativement différents entre eux et supérieurs de $10 \%$ et de $36 \%$ à ceux des moutons.

Enfin, l'effet de l'aliment concentré a été important : baisse significative de $30 \%$ (17 $\mathrm{min}$ ) de la durée des grands repas de fourrage.

\section{Quantités ingérées et durée unitaire d'ingestion lors des grands repas}

Les quantités moyennes de MS de fourrage ingérées lors des grands repas du matin figurent, dans le tableau IX. Elles représentent 30 à $34 \%$ des quantités de fourrage ingérées par jour.

Les durées unitaires d'ingestion, lors de ces grands repas, sont égales à $4,50 \mathrm{~min} /$ $\left(\mathrm{g} / \mathrm{kg} \mathrm{P}^{0,75}\right.$ ) contre 4,70 au niveau de l'ensemble des repas. La différence n'est pas significative. Ces durées unitaires ont été comparables pour l'ensilage de maîs et le foin de ray-grass (en moyenne $3,97 \mathrm{~min} /$ $\left(\mathrm{g} / \mathrm{kg} \mathrm{P}^{0,75}\right)$ et plus élevées pour le foin de fléole (5,38 $\mathrm{min} /(\mathrm{g} / \mathrm{kg} \mathrm{P0,75})$.

Pour les fourrages étudiés, béliers et brebis donnent des résultats comparables. Les chèvres ont une durée unitaire d'ingestion supérieure de $7,5 \%$ à celles des moutons et les vaches une durée inférieure de $23 \%$.

Enfin, lorsque ces animaux reçoivent de l'aliment concentré, le paramètre étudié augmente de $10 \%$ (vitesse d'ingestion plus faible).

\section{Activités de rumination (tableaux VI, VII, VIII)}

\section{Durée journalière}

Par rapport aux paramètres présentés plus haut, la durée journalière de rumination ne varie que modérément d'un individu à l'autre dans un lot : coefficient de variation de $8,5 \%$ pour les vaches, 12 pour les brebis, 13 pour les béliers, 15 pour les chèvres et les taurillons. 
Tableau IX. Quantités de MS de fourrage ingérées et durée unitaire d'ingestion lors des grands repas du matin -6 régimes - (ensilages de maïs +2 foins).

\section{Moutons Brebis Chèvres Taurillons Vaches}

$\begin{array}{lccccc}\text { Quantités ingérées (g MS/kg P0,75) } & 17,1 & 14,9 & 15,3 & 20,8 & 29,7 \\ \text { Durée unitaire d'ingestion (min/g/kg } \mathrm{P}^{0,75} \text { ) } & 4,85 & 4,75 & 5,21 & 3,79 & 3,72 \\ \text { Proportion de fourrage ingérée } & 34 & 30 & 30 & 32 & 31\end{array}$

Les valeurs obtenues pour les différents fourrages varient entre $445 \mathrm{~min}$ pour l'ensilage de maïs, 487 pour l'ensilage d'herbe, 518 pour le foin de fléole et 553 pour le foin de ray-grass.

Les béliers et les vaches ont ruminé pendant $510 \mathrm{~min} / \mathrm{j}$. Cette valeur a été plus faible de $5 \%(S)^{*}$ pour les brebis, de $23 \%$ (S) pour les chèvres et de $26 \%$ (S) pour les taurillons.

L'apport d'aliment concentré a fait baisser significativement la durée journalière de rumination de $7,2 \%(35 \mathrm{~min} / \mathrm{j})$.

\section{Durée unitaire}

Le coefficient de variation de ce paramètre varie assez nettement d'un animal à l'autre: de $8 \%$ pour les vaches à $12 \%$ pour les béliers, $13 \%$ pour les brebis, $14 \%$ pour les taurillons et $21 \%$ pour les chèvres.

Les valeurs trouvées pour les différents fourrages sont égales respectivement à $7,68 \mathrm{~min} /\left(\mathrm{g} / \mathrm{kg} \mathrm{P}^{0,75}\right)$ pour l'ensilage de maïs, 8,83 pour le foin de ray-grass, 9,90 pour le foin de fléole et 7,17 pour l'ensilage d'herbe.

Ce sont les béliers qui ont mis le plus de temps à ruminer par $\mathrm{g}$ de MS ingérée (10 $\mathrm{min} /(\mathrm{g} / \mathrm{kg} \mathrm{P0,75})$. Les valeurs enregistrées sont plus faibles de $3 \%$ pour les bre- bis (NS), $17 \%$ pour les chèvres (S), $44 \%$ pour les taurillons (S) et de $46 \%$ pour les vaches (S).

Nous avons considéré que l'aliment concentré ne nécessitait pas de rumination. On note cependant que le paramètre étudié augmente de $12 \%$ lorsque les animaux reçoivent ce concentré.

\section{Temps de latence}

Entre la fin de chaque grand repas et le début de la rumination il existe un temps de latence que nous avons systématiquement mesuré. La variabilité de ce paramètre est extrêmement grande : coefficients de variation compris entre 36 et $46 \%$.

L'effet du type de fourrage est important : 87 min pour l'ensilage de maïs, 28 pour le foin de ray-grass, 22 pour le foin de fléole et 51 pour l'ensilage d'herbe.

II y a aussi de grandes différences entre les types d'animaux. Béliers et brebis ont le même temps de latence, mais ce temps augmente de $44 \%$ chez les chèvres (S) et il baisse de $29 \%$ chez les taurillons (S) et de $54 \%$ chez les vaches (S).

Enfin, l'apport de concentré augmente significativement de $45 \%$ ce temps de latence (+ $18 \mathrm{~min})$. 


\section{Activités de mastication}

\section{Durée journalière}

La variabilité de ce paramètre est relativement faible et comparable à celle des quantités journalières ingérées : coefficient de variation intralot de $11,7 \%$ pour les béliers, $9,8 \%$ pour les brebis, $11,5 \%$ pour les chèvres, $10,8 \%$ pour les taurillons et $7,2 \%$ pour les vaches.

En moyenne, les valeurs pour les foins sont comparables : $825 \mathrm{~min} / \mathrm{j}$ pour le raygrass et 849 pour la fléole. Ces valeurs sont beaucoup plus faibles pour les ensilages : $675 \mathrm{~min} / \mathrm{j}$ pour l'ensilage de maïs et 710 pour l'ensilage d'herbe.

Les béliers et les brebis ont passé le même temps à mastiquer. Les vaches laitières ont passé $17 \%$ de temps en plus $(S)$, mais les chèvres $12 \%$ en moins $(S)$ et les taurillons $10 \%(\mathrm{~S})$.

Enfin, lorsque les régimes comportaient de l'aliment concentré, la durée journalière de mastication a diminué significativement de $13 \%$.

\section{Durée unitaire}

Les coefficients de variation de ce paramètre vont de $9 \%$ pour les vaches et les béliers à $11 \%$ pour les taurillons, $13 \%$ pour les brebis et $16 \%$ pour les chèvres. II y a de gros écarts entre fourrages, les valeurs moyennes étant respectivement égales à $11,32 \mathrm{~min} /(\mathrm{g} / \mathrm{kg} \mathrm{P0,75}$ ) pour l'ensilage de maïs, 13,02 pour le foin de ray-grass, 16,00 pour le foin de fléole et 10,23 pour l'ensilage d'herbe.

Les résultats obtenus pour les béliers et les brebis sont comparables $(+2,5 \%$ seulement pour les brebis par rapport aux béliers). Les chèvres passent $4,5 \%$ de leur temps en moins (NS) que les béliers à mastiquer $1 \mathrm{~g}$ de MS ingéré, les taurillons $32 \%$ en moins (S) et les vaches $36 \%$ (S).
Enfin, l'apport d'aliment concentré a un léger effet dépressif sur l'efficacité de la mastication du fourrage car il augmente de $5,6 \%$ le temps passé à mastiquer $1 \mathrm{~g}$ de MS ingéré/kg $P^{0,75}$.

\section{DISCUSSION}

\section{Quantités ingérées}

Avant de discuter les résultats concernant les activités alimentaires et méryciques des animaux il est nécessaire de savoir quelle est la validité des quantités ingérées enregistrées.

D'après Bocquier et al (1988), les brebis taries pesant $60 \mathrm{~kg}$, ont une capacité d'ingestion qui décroît de 1,9 à 1,6 UEM lorsque leur note d'état physiologique passe de 2 (maigres) à 4 (grasses). Les brebis utilisées ici étaient plutôt maigres. Elles auraient donc dû ingérer un peu plus que les moutons dont la capacité d'ingestion théorique est de 1,62 UEM pour le même poids (Demarquilly et al, 1978). Cela a effectivement été le cas avec l'ensilage de maïs, mais pas avec les autres fourrages. II est possible que le maintien des brebis dans les cages à métabolisme ait limité leur appétit, de plus, étant maigres au début de chaque période, elles se sont engraissées rapidement, ce qui a dû concourir aussi à limiter leur appétit. Finalement, la comparaison des activités alimentaires des brebis et des béliers castrés est intéressante puisqu'elle est effectuée à même niveau d'ingestion.

Dans nos essais, les chèvres taries ont ingéré un peu moins que les ovins. Geoffroy (1974), pour 5 rations, trouve $56 \mathrm{~g}$ de $\mathrm{MS} / \mathrm{kg} \mathrm{P0,75}$ pour les chèvres contre 60 pour les béliers (différence non significative), soit une tendance comparable à la 
nôtre. D'autres essais vont dans ce sens (Quick et Dehority, 1986; Masson et al, 1986) alors que Mc Cabe et Barry (1988) et Howe et al (1988) trouvent l'inverse. A partir d'une revue bibliographique, Brown et Johnson (1984) considèrent cependant que les moutons ingèrent plus que les chèvres, sauf pour les fourrages de mauvaise qualité. Howe et al (1988) concluent que les quantités ingérées de ces espèces sont comparables pour les fourrages classiques, les chèvres ingérant beaucoup mieux les feuillages. Finalement, la tendance que nous avons enregistrée n'est pas en désaccord avec la bibliographie. Elle pourrait cependant être liée aussi aux contraintes imposées aux chèvres pour les mesures (Mac Farlane, 1982).

D'après Geay et Micol (1988) les taurillons utilisés ont une capacité d'ingestion de 6,6 à 7,0 UEB. Nos calculs donnent des valeurs d'environ $7,6 \mathrm{UEB}$, ce qui montre que les animaux utilisés ont ingéré plus que prévu.

Enfin d'après Hoden et al (1988), les vaches auraient une capacité d'ingestion de 14,8 UEL. Nous trouvons 13,6 UEL, ce qui, à l'inverse de ce qui est observé sur taurillons, est faible et dû probablement à l'état d'engraissement relativement élevé des animaux utilisés.

Les taux de substitution varient dans le même sens que l'excès des apports énergétiques par rapport aux besoins (Faverdin et al, 1990). Il y a donc cohérence avec cette théorie pour les 4 fourrages. En revanche, par rapport à ce qu'on trouve habituellement (Dulphy et al, 1987), le taux de substitution est plutôt faible dans nos essais pour les béliers et plus élevé pour les taurillons.

II existe donc quelques divergences entre les quantités ingérées habituellement et celles trouvées pour les rations étudiées. Ceci n'est cependant pas trop grave dans la mesure où les activités alimentaires et méryciques observées ne sont discutées que par rapport aux quantités ingérées réellement obtenues.

Les quantités ingérées présentées sont calculées par rapport au poids métabolique (PV0,75) tel qu'il est classiquement défini. II est admis que la définition retenue est adaptée à la comparaison des animaux d'un même type. II nous faudra vérifier la validité de ce mode d'expression du poids lorsqu'on compare des animaux d'espèces différentes. Ce point sera discuté dans la deuxième partie de ce travail ayant trait en particulier à l'influence du poids vif sur les paramètres enregistrés.

Enfin, il faut souligner un autre point concernant les quantités ingérées, ce sont les variations individuelles. On observe une tendance assez nette à ce que ces variations soient faibles pour les vaches et élevées pour les petits ruminants. Cela traduit des différences d'homogénéité des animaux, liées probablement à leur sélection plus ou moins poussée. Pour les vaches, nous observons un coefficient de variation de $6,8 \%$, proche de celui trouvé par Dulphy et al (1989), soit 6,6\% et Faverdin et al (1987), soit $7,8 \%$. Nous avions trouvé $8,5 \%$ pour les génisses et obtenons $8,3 \%$ dans ce travail pour les taurillons. Les valeurs pour les béliers et les chèvres sont identiques. Cependant, le nombre de chèvres conservées par lot a été un peu plus faible que pour les béliers (en moyenne 4,83 contre 5,37). La valeur moyenne de $13,7 \%$ pour les brebis est en revanche très élevée ( 5 brebis conservées par lot).

\section{Répartition des activités alimentaires}

II y avait 2 distributions d'aliments par jour, suivies à chaque fois d'un grand repas. II n'est donc pas possible d'étudier une ré- 
partition spontanée des activités. On constate cependant qu'après une distribution de fourrage, les animaux "stockent" environ $30 \%$ de ce qu'ils ingèrent chaque jour dans leur rumen. Ils le font avec une vitesse d'ingestion un peu plus élevée que celle observée lors des petits repas. A ce niveau, les différences entre types d'animaux sont relativement faibles. Seules, les brebis ont tendance à ingérer un peu moins lors de ces grands repas. Environ $40 \%$ du fourrage sont ensuite ingérés en 4 à 7 petits repas, ayant donc chacun un faible volume. A ce niveau aussi les différences entre types d'animaux sont plutôt faibles, seuls les béliers castrés ayant tendance à faire un peu moins de petits repas.

Déjà Geoffroy (1974) puis Masson et al (1989) avaient observé moins de repas chez le mouton que chez la chèvre. Les bovins aussi semblent faire plus de petits repas que les moutons (Dulphy et al, 1984 avec des ensilages d'herbe, Dulphy et Michalet-Doreau, 1983, avec des fourrages verts). Finalement, les animaux utilisés ici ne peuvent pas stocker en une seule fois leur nourriture. Cela rejoint bien le fait qu'au pâturage il y a 2 pics d'ingestion par jour (Dulphy et al, 1979). Cela est vrai également si on distribue le fourrage en une seule fois à l'auge (Dulphy et al, 1988).

Le nombre de repas par jour, la durée des grands repas et la vitesse d'ingestion lors de ces grands repas sont des paramètres très variables et il $y$ a souvent plus de différences entre individus d'une même espèce qu'entre espèces. Cela veut dire qu'en dehors du schéma général - un grand repas après chaque distribution d'aliment, puis un nombre réduit de petits repas - les "profils alimentaires" peuvent être très variables entre animaux ou entre espèces, même lorsqu'il n'y a pas de différences de quantités ingérées. Cela signifie aussi que la précision des valeurs des pa- ramètres descriptifs utilisés est faible (coefficient de variation de l'ordre de 13 à $19 \%)$.

\section{Durée et vitesse d'ingestion}

D'un point de vue global, la durée journalière d'ingestion détermine directement, avec la vitesse d'ingestion, les quantités ingérées.

Le temps passé à ingérer pour les ovins et les caprins, qui sont à l'entretien, représente, dans cette étude, de 15 à $17 \%$ du temps journalier. Pour des quantités ingérées comparables, les brebis ont ingéré plus longtemps, donc moins vite que les béliers castrés. Pour des quantités ingérées légèrement plus faibles, les chèvres ont ingéré à la fois plus longtemps et donc encore moins vite que les béliers. Ruckebush et Bost (1963), ainsi que Geoffroy (1974) et Focant (1986) avaient déjà observé pour la brebis, une prise de nourriture plus rapide que celle de la chèvre. Ces observations traduisent en termes quantitatifs la constatation classique que la chèvre sélectionne plus que les autres ruminants ce qu'elle ingère (Morand-Fehr et al, 1980; Van Soest, 1982). Masson et al (1986) observent la même chose lorsque ces espèces reçoivent de la paille. Cependant, cette constatation n'est peut-être pas généralisable car ces derniers auteurs, pour un foin, ainsi que Masson et al (1989) pour 2 pailles, observent que les boucs ingèrent plus rapidement que les béliers.

Dans notre étude, les bovins ingèrent plus longtemps que les petits ruminants, mais ils ont des besoins de production et il est probable que cela joue autant, voire plus, que l'espèce animale, pour expliquer ce qui est observé $(20 \%$ du temps journalier pour les taurillons, 23\% pour les vaches). Les résultats récapitulés par Jarrige (1978) sont en général plus élevés, 20 à $39 \%$ du temps journalier pour des 
vaches en lactation, donc produisant plus que celles utilisées ici, mais recevant des fourrages plus diversifiés.

Les coefficients de variation intralot de ces 2 paramètres sont légèrement plus faibles que ceux décrivant la répartition des activités alimentaires : en moyenne 12 à $13 \%$. Leur rôle est donc probablement plus important que celui du nombre et de la répartition des repas pour expliquer les différences individuelles de quantités ingérées.

\section{Activités de rumination}

La plage de variation observée sur le temps de rumination est très élevée : minimum de $330 \mathrm{~min}$ pour les chèvres recevant l'ensilage d'herbe et l'aliment concentré; maximum de 580 min pour les vaches recevant l'ensilage d'herbe seul. Pour les ovins, nous avions émis l'hypothèse (Dulphy et Béchet, 1976) qu'il existait une limite située vers $550-600 \mathrm{~min} / \mathrm{j}$. II en est d'ailleurs peut-être de même pour les vaches laitières dont la durée de rumination varie très peu après le vélage alors que les quantités ingérées augmentent nettement (Doreau et Rémond, 1982). Dans nos essais, à part pour le foin de ray-grass (cas des moutons, des brebis et des vaches) cette limite n'est pas atteinte. Il est donc probable qu'ici, la durée de rumination a été une conséquence du niveau d'ingestion et n'a pas limité les quantités ingérées. Par ailleurs, les différences entre types d'animaux semblent plus dépendantes du temps passé à ingérer : si ce temps augmente, la durée de rumination a tendance à diminuer.

Après un grand repas, les bovins ont tendance à commencer leur rumination plus rapidement que les petits ruminants. L'explication est peut-être à rechercher dans des différences anatomiques induisant des différences d'intensité des stimu- lations dues au contenu ruminal. En effet, les piliers du rumen sont plus marqués chez les bovins (Florentin, 1952) que chez les ovins.

Avec les ensilages (maïs et herbe), le temps de latence augmente nettement. Leur structure moins fibreuse que celle des foins stimule probablement moins la rumination. L'apport de concentré doit diminuer également l'effet stimulant des fourrages.

\section{Activités de mastication}

Compte tenu des compensations qui peuvent exister entre les durées d'ingestion et de rumination, la somme de ces durées est probablement plus intéressante à considérer, d'autant plus que c'est avant tout la mastication, quelle que soit sa nature, qui permet la réduction des aliments en fines particules.

Au niveau de cette durée de mastication, il existe des différences entre types d'animaux, les chèvres et les taurillons présentant des valeurs plutôt faibles et les vaches une valeur plutôt élevée.

En ce qui concerne le temps passé à mastiquer une même quantité, moutons et brebis ont la même efficacité. Les chèvres semblent un peu plus efficaces $(1 \mathrm{~min}$ de moins par $\mathrm{g} / \mathrm{kg} P \mathrm{PV}^{0,75}$ ). En fait, elles mastiquent $1,57 \mathrm{~g}$ de $\mathrm{MS} / \mathrm{min}$ contre 1,85 pour les ovins, ceci parce qu'elles sont plus légères. Là aussi se pose donc le problème de l'exposant du poids vif à choisir pour comparer des espèces de poids différent. Masson et al (1986) trouvent la même tendance avec du foin, mais pas avec de la paille traitée. Masson et al (1989) observent une valeur plus faible de $2,5 \mathrm{~min} /(\mathrm{g} / \mathrm{kg}$ $\mathrm{P}^{0,75}$ ) pour des boucs par rapport à des béliers de poids comparables. Les taurillons passent 3 à 5 min de moins à mastiquer par $\mathrm{g} / \mathrm{kg} P V 0.75$. Ils mastiquent en 
moyenne $10,07 \mathrm{~g}$ de $\mathrm{MS} / \mathrm{min}$. Les vaches laitières mastiquent $16,5 \mathrm{~g}$ de $\mathrm{MS} / \mathrm{min}$. Pour comparer objectivement les espèces, il faudrait peut-être corriger ces valeurs par la surface de la table dentaire. Finalement, plus que l'espèce, c'est très probablement le format de l'animal qui joue un rôle, à la fois par les différences de taille des mâchoires et les différences de taille de l'orifice réticulo-omasal permettant, chez les plus grands, à des particules plus grandes de sortir du rumen (Ulyatt et al, 1986).

Si on examine les parts respectives des temps d'ingestion et de rumination dans la mastication on discrimine 2 groupes : chèvres, taurillons et vaches passent $40 \%$ de leur temps de mastication à ingérer contre seulement $30 \%$ pour les ovins. Les ovins ingèrent donc relativement vite, comme cela a déjà été dit, et ils doivent compenser en ruminant relativement plus longtemps.

D'une espèce à l'autre, malgré tout, alors que les quantités ingérées en $\mathrm{kg} / \mathrm{j}$ varient de 1 (chèvres) à 14 (vaches), la durée de mastication n'augmente que de $33 \%$. C'est donc bien le paramètre $\mathrm{g} / \mathrm{min}$ ou $\mathrm{min} / \mathrm{g}$ qui est le plus discriminant entre types d'animaux et probablement d'un type de fourrage à l'autre (Dulphy et al, 1979).

\section{CONCLUSION}

Le travail présenté ici reste très global car de nombreuses mesures, d'ordre plus physiologiques font défaut pour interpréter ce qui a été observé. Quelques conclusions peuvent néanmoins en être tirées :

- 1) pour arriver à un niveau d'ingestion donné les différents individus d'un groupe et les différentes espèces peuvent organiser de façon très différente leur emploi du temps :

- part variable des grands repas;
- nombre variable des petits repas;

- ingestion plus ou moins rapide, compensée, si nécessaire, par une durée de rumi.nation plus longue.

II existe bien des différences entre espèces, mais ce travail souligne les différences importantes entre individus.

- 2) Alors que les quantités ingérées varient pour les espèces étudiées, de 1 à 14 , la durée journalière de mastication n'augmente que de $33 \%$. Dans tous les cas, la durée de mastication étant élevée, elle ne peut donc pas varier beaucoup d'une espèce à l'autre.

- 3) Ces particularités : faible variation des durées de mastication, souplesse relative dans la répartition de ces activités de mastication, face à des quantités ingérées très différentes, devraient aider à mieux comprendre quels sont les facteurs qui régulent les quantités ingérées dans le court terme.

En effet, le moment de la fin du grand repas, puis ceux du début et de la fin des petits repas sont influencés par l'état de réplétion du réticulo-rumen (Dulphy et Faverdin, 1987) et les signaux qu'il génère (Forbes, 1980). L'action de ces signaux peut être modulée par les besoins ou l'état de l'animal. Le comportement des animaux est donc le reflet de phénomènes physiologiques qui peuvent ensuite être étudiés par des méthodologies plus spécifiques. L'enregistrement de ce comportement est alors probablement indispensable pour repérer les événements déterminant cette régulation des quantités ingérées. La comparaison d'individus et d'espèces est sûrement tout aussi importante que celle de fourrages différents.

- 4) II est apparu dans la discussion des résultats des petites difficultés car, en fait, on a comparé à la fois des espèces et des situations différentes (format, besoins, mode d'élevage, etc.). 
En effet, on est tenté a priori de prendre les animaux utilisés et déjà bien connus présents sur un site de recherche, comme nous l'avons fait. II faudrait donc aller plus loin et comparer des espèces préparées dans des conditions plus strictes et longtemps à l'avance (Brown et Johnson, 1984).

Enfin, la relation entre les différents paramètres, la façon dont interviennent le poids vif des animaux, les quantités ingérées, la digestibilité éventuellement, sont à examiner pour aller plus loin. Nous nous y attacherons ultérieurement.

\section{RÉFÉRENCES}

Bocquier $F$, Theriez $M$, Prache $S$, Brelurut $A$ (1988) Alimentation des ovins. In: Alimentation des bovins, ovins, caprins. INRA Publ, 249-281

Brown LE, Johnson WL (1984) Comparative intake and digestibility of forages and byproducts by goats and sheep: a review. Int Goat Sheep Res 2 (3), 212-226

Carle B, Dulphy JP (1980) Comportement alimentaire comparé des ovins et des bovins. Relation avec la digestion des aliments. Reprod Nutr Dév 20, 1633-1639

Chenost M, Martin-Rosset W (1985) Comparaison entre espèces (mouton, cheval, bovin) de la digestibilité et des quantités ingérées des fourrages verts. Ann Zootech 34, 291 312

Conrad HR, Pratt AD, Hibbs JW (1964) Regulation of feed intake in dairy cows. I - Change in importance of physical and physiological factors with increasing digestibility. $J$ Dairy Sci 47, 54-67

Demarquilly C, Andrieu J, Sauvant D (1978) Composition et valeur nutritive des aliments. In: Alimentation des Ruminants. INRA Publ, Versailles, $597 p$

Doreau M, Rémond B (1982) Comportement alimentaire et utilisation digestive d'une ration de composition constante chez la vache laitière en fin de gestation et début de lactation. Reprod Nutr Dév 22, 307-324
Dulphy JP (1971) Influence du poids vif et du niveau d'ingestion sur le comportement alimentaire et mérycique du mouton. Ann Zootech $20,477-486$

Dulphy JP, Bechet G (1976) Influence du stade de végétation et de l'espèce végétale sur le comportement alimentaire et mérycique de moutons recevant des fourrages verts hachés. Ann Zootech 25, 505-519

Dulphy JP, Demarquilly C (1981) Problèmes particuliers aux ensilages. In: Prévision de la valeur nutritive des aliments des ruminants. INRA Publ, Versailles, 81-104

Dulphy JP, Michalet-Doreau B (1983) Comportement alimentaire et mérycique d'ovins et de bovins recevant des fourrages verts. Ann Zootech 32, 465-474

Dulphy JP, Carle B (1986) Activités alimentaires et méryciques comparées des bovins, des caprins et des ovins. Reprod Nutr Dév 26, 279-280

Dulphy JP, Faverdin P (1987) L'ingestion alimentaire chez les ruminants : modalités et phénomènes associés. Reprod Nutr Dév 27, 129-155

Dulphy JP, Remond B, Thériez M (1979) Ingestive behaviour and related activities in ruminants. In: Digestive Physiology and Metabolism in Ruminants ( $\mathrm{Y}$ Ruckebush, $\mathrm{P}$ Thivend, eds) MPT Press Ltd, 103-122

Dulphy JP, Michalet-Doreau B, Demarquilly C (1984) Étude comparée des quantités ingérées et du comportement alimentaire et mérycique d'ovins et de bovins recevant des ensilages d'herbe réalisés selon différentes techniques. Ann Zootech 33, 291-320

Dulphy JP, Faverdin P, Micol D, Bocquier F (1987) Révision du système des Unités d'Encombrement (UE). Bull Tech CRVZ Theix, INRA 70, 35-48

Dulphy JP, El Meddah Y, Baumont R (1988) Influence du rythme de distribution sur les activités alimentaires et l'évolution journalière du contenu ruminal chez le mouton. Reprod Nutr Dév 28, 919-929

Dulphy JP, Demarquilly C, Remond B (1989) Quantités d'herbe ingérées par les vaches laitières, les génisses et les moutons : effet de quelques facteurs de variation et comparaison entre ces types d'animaux. Ann Zootech 38, 107-119 
Ellis WC, Wylie MI, Matis JH (1988) Dietary digestive interactions determining the feeding value of forages and roughages. In: World Animal Science Series, Vol B-4, Elsevier, Amsterdam, 177-229

Faverdin P, Hoden A, Coulon JB (1987) Recommandations alimentaires pour les vaches laitières. Bull Tech CRZV Theix INRA, 133-152

Faverdin $P$, Dulphy JP, Coulon JB, Vérité $R$ Garel JP, Rouel J, Marquis B (1990) Substitution of roughage by concentrates for dairy cows. Livest Prod Sci (sous presse)

Florentin $P$ (1952) Mise au point sur la situation et les voies de communication intérieures des réservoirs gastriques chez les ruminants domestiques. Rev Méd Vét 103, 530-542

Focant $M$ (1984) Comportement alimentaire, rumination, fermentations réticulo-ruminales et acides gras volatils plasmatiques, comparés chez la chèvre et le mouton; influence du régime. Reprod Nutr Dév 24, 229-250

Focant M (1986) Contribution à l'étude du comportement alimentaire des petits ruminants : rôle des acides gras volatils. Thèse Université catholique de Louvain. $262 p$

Forbes JM (1980) A model of short-term control of feeding in the ruminant : effects of changing animal or feed characteristics. Appetite 1, 21-41

Geay Y, Micol D (1988) Alimentation des bovins en croissance et à l'engrais. In: Alimentation des bovins, ovins et caprins. INRA Publ 213248

Geoffroy $F$ (1974) Étude comparée du comportement alimentaire et mérycique de deux petits ruminants : la chèvre et le mouton. Ann Zootech 23, 63-73

Hoden A, Coulon JB, Faverdin P (1988) Alimentation des vaches laitières. In: Alimentation des bovins, ovins et caprins. INRA Publ 135158

Howe JC, Barry TN, Popay Al (1988) Voluntary intake and digestion of gorse (Ulex europaeus) by goats and sheep. J Agric Sci Camb 111, 107-114

Huston JE, Engdahl BS, Bales KW (1986) Intake and digestibility in sheep and goats fed three forages with different levels of supplemental protein. Small Ruminant Res 1, 81-92

Jarrige R (1978) Consommation d'aliments et d'eau. In: Alimentation des Ruminants. INRA Publ, Versailles, 177-206
Mac Farlane WV (1982) Concepts in animal adaptation. In: Proc III Int Conf Goat Prod Disease. Dairy Goat Journal Publ C, Scottsdale, AZ 375-385

Masson C, Alrahmoun W, Tisserand JL (1986) Étude comparée de la quantité ingérée, de la digestibilité, de l'utilisation de l'azote, du temps moyen de rétention et du comportement alimentaire chez les jeunes caprins et ovins recevant différents régimes. Ann Zootech 35, 49-60

Masson C, Kirilov D, Faurie F, Tisserand JL (1989) Comparaison des activités alimentaires et méryciques d'ovins et de caprins recevant de la paille d'orge traitée ou non à la soude. Ann Zootech 38, 73-82

Mc Cabe Sharon M, Barry TN (1988) Nutritive value of willow (Salix $s p$ ) for sheep, goats and deer. J Agric Sci Camb 111, 1-9

Morand-Fehr P, Hervieu J, Sauvant D (1980) Contribution à la description de la prise alimentaire de la chèvre. Reprod Nutr Dév 20, 1641-1644

Quick TC, Dehority BA (1986) A comparative study of feeding behaviour and digestive function in dairy goats, wool, sheep and hair sheep. J Anim Sci 63, 1516-1526

Ruckebush $Y$ (1963) Recherches sur la régulation centrale du comportement alimentaire chez les ruminants. Thèse Doc Sci Nat Univ Lyon, $213 \mathrm{p}$

Ruckebush Y, Bost J (1963) Étude comparée de la motricité du réticulum et du comportement alimentaire chez les ovins et les caprins en stabulation. Rev Med Vet CXIV 3, 184-195

Thivend P, Mercier C, Guilbot A (1965) Dosage de l'amidon dans les milieux complexes. Ann Biol Anim Biochim Biophys 5, 513-526

Ulyatt MJ, Dellow DW, John A, Reid CSW, Waghorn GC (1986) Contribution of chewing during eating and rumination to the clearance of digesta from the reticulo-rumen. In: Control of Digestion and Metabolism in Ruminants (LP Milligan, WL Growum, A Dobson, eds) Prentice Hall, Englewood Clifts, New Jersey, USA, 498-515

Van Soest PJ (1982) Nutritional Ecology of the Ruminants. $\mathrm{O}$ and $\mathrm{B}$ Brooks Inc, Corvallis, Oregon 